\title{
Priority-driven Self-optimizing Power Control Scheme for Interlinking Converters of Hybrid AC/DC Microgrid Clusters in Decentralized Manner
}

\author{
Xiaochao Hou, Member, IEEE, Kai Sun, Senior Member, IEEE, Ning Zhang, Senior Member, IEEE, \\ Fei Teng, Senior Member, IEEE, Xin Zhang, Senior Member, IEEE, and Tim C. Green, Fellow, IEEE
}

\begin{abstract}
${ }^{1}$ Abstract-Hybrid AC/DC microgrid clusters are key building blocks of smart grid to support sustainable and resilient urban power systems. Practically, in networked microgrid clusters, the subgrid load-priorities and power quality requirements for different areas vary significantly. In order to realize optimal power exchanges among microgrid clusters, this study proposes a decentralized self-optimizing power control scheme for interlinking converters (ILCs). First, a prioritydriven optimal power exchange model of ILCs is built that fully considers the priorities and capacities in subgrids. The whole optimization objective is to minimize the total DC-voltage/ACfrequency state deviations of subgrids. Second, to realize the decentralized power flow control, an optimal-oriented quasidroop control strategy of ILCs is introduced. Consequently, as each of ILCs only monitors the local AC-side frequency and DCside voltage signals, the whole optimal power control of the wide-area microgrid clusters is achieved in a decentralized manner without any communication link. Thus, the proposed control algorithm has the features of decreased cost, increased scalability, reduced geographic restrictions and high resilience and robustness in terms of communication faults. Finally, the proposed method is validated by three cases in hardware-in-loop (HIL) environment.
\end{abstract}

Keywords-Decentralized self-optimizing, droop control, hybrid microgrid, interlinking converter, networked microgrids.

\section{INTRODUCTION}

Microgrids have become a cost-efficient solution to locally integrate the high penetration of distributed generations (DGs) and provide reliable power to customers in future urban power systems [1]. It can maintain the emergent operations and protect critical infrastructure from power outages in the event of physical/cyber disruptions in the utility distribution grid [2].

According to the voltage type of bus supply, microgrids can be classified into three categories: AC microgrid, DC microgrid, and hybrid AC/DC microgrid [3]-[4]. In the early decades, AC microgrid may dominate existing distribution

${ }^{1}$ Manuscript received August 1, 2021; revised October 17, 2021; accepted November 9, 2021. This work was supported in part by the National Natural Science Foundations of China under Grants 52061635101 and 61733010, the China Postdoctoral Science Foundation under Grant 2020M680553, the Delta Power Electronics Research and Education Development Program under Grant DREM2019005. (Corresponding author: Kai Sun.)

X. Hou, K. Sun, and N. Zhang are with the State Key Lab of Power Systems, Department of Electrical Engineering, Tsinghua University, Beijing 100084, China (e-mail: houxiaochao@tsinghua.edu.cn; sun-kai@mail. tsinghua.edu.cn; ningzhang@tsinghua.edu.cn).

F. Teng and T. Green are with the Department of Electrical and Electronic Engineering, Imperial College London, London SW7 2AZ, U.K. (e-mail: f.teng@imperial.ac.uk; t.green@imperial.ac.uk).

X. Zhang is with the College of Electrical Engineering, Zhejiang University, Hangzhou 310027, China (e-mail: zhangxin_ieee@zju.edu.cn). systems with connecting distributed micro-sources or forming an islanded system [5]. But, recently, with the advancement of DC technologies and increased DC sources/loads, DC microgrid has been a promising structure due to the features of high efficiency and free of harmonics/reactive power. Especially, the DC sources, such as photovoltaic, fuel cell and storage, are directly connected to DC microgrid to avoid the loss of DC-AC conversions [6].

In addition, interlinking converters (ILCs) are implemented to interlink the DC microgrid and AC microgrid together to form a hybrid microgrid [7], which has been regarded as the most likely future microgrid structures by integrating the advantages of both $\mathrm{AC}$ and DC microgrid [8]. As DC and AC sources/loads are allocated in their DC and AC respective subgrids, the power conversions are greatly reduced, and the system has the increased efficiency [9].

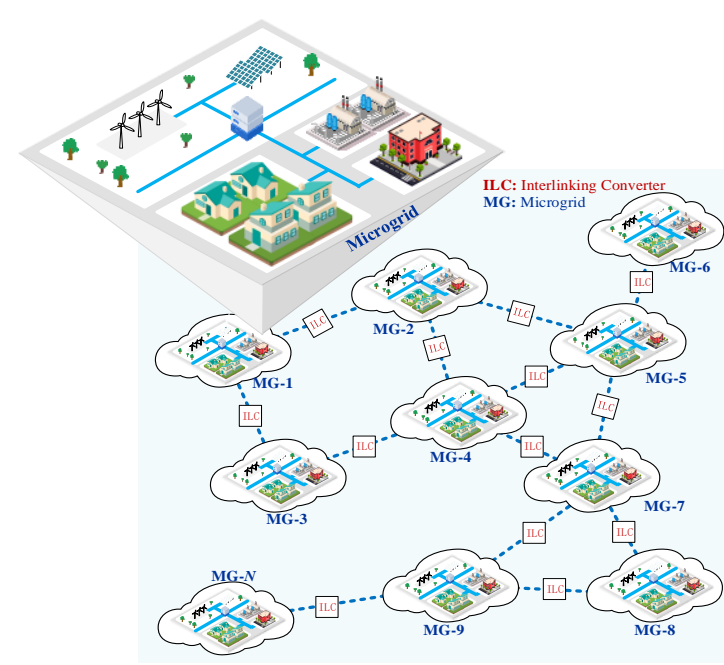

Fig. 1. Schematic representation of networked microgrid clusters.

Networked hybrid microgrid clusters are formed by interlinking the microgrids with their individual neighbors through ILCs, as shown in Fig. 1. Rather than focusing on a single microgrid, multiple microgrid clusters can further enhance the systematic and coordinated operation [10]. A single microgrid is expected to feed its local loads independently, however, due to the intermittency of wind/ solar-based renewable generations and the load uncertainty, it is likely that the microgrid experiences power deficiency. This problem can be mitigated by coupling the overloaded microgrid to other neighbor microgrids that have power surplus. Considering the sharing and supporting advantages of 
networked microgrids, it is still a fundamental challenge to control and optimize the multiple functions of ILCs [11].

In hybrid microgrid clusters, the role and control methods of ILCs significantly affect the optimal power exchange [12], power quality [13]-[15], system stability [16], resilience capability [17], and ancillary services [18]-[20]. In [12], the power hub functions of the ILC in networked microgrids are comprehensively reviewed and discussed to enhance the grid resilience, robustness, and efficiency. In [13]-[15], the power quality improvement methods of the ILC are proposed on the harmonic and unbalance conditions. In [16], the ILC serves as active stabilizations to provide stability damping among microgrids. For the resilience enhancement, a flexible division and unification control method is proposed in [17]. With regard to ancillary services, a virtual synchronous generator characteristic is imitated for the ILC to provide inertia support in [18]. Considering the flexibility and compatibility of multioperation modes, two distributed uniform control strategies are developed for the ILC [19]-[20]. Although the aforementioned research promotes the multi-functional works of ILCs, they are mostly suitable for the hybrid microgrid with two symmetrical subgrids. When applied in the hybrid microgrid clusters with different priorities and capacities, it leads to some limitations because of the different requirements of power quality, stability region and fault-tolerance in each subgrid. That is, the power supply of critical subgrids with high-priority should be guaranteed even if have to sacrifice other subgrids with low-priority. Thus, the control function design of ILCs in microgrid clusters is more complicated from the system-wide optimal viewpoint.

With regard to the control framework, the power control strategies of ILCs are classified into three categories: centralized [21]-[23], distributed [24]-[26] and decentralized [27]-[33], according to the communication dependency. In the central communication-based methods, the demand response [21], load shedding [22], and energy management [23] can be easily achieved by high-bandwidth communication network for mass information exchange within microgrid clusters. However, the central controller processor has the single-pointfailure problem and has significant computational burden. As the number of distributed generations and microgrids increase, the computational complexity increases exponentially, which largely decreases the reliability and limits the system scale. Then, distributed communication-based methods are proposed by avoiding a central station [24]-[26]. Although agents work autonomously in a cooperative way to reach a global control objective, a tight neighbor communication network is required for information sharing, and thus the communicationdepended delay, packet-loss, and failure problems decreases system stability, reliability and resilience.

Presently, decentralized power control of the ILC has been increasingly studied in [27]-[33] and provides an alternative solution to manage complex microgrid structures without communication. In [27]-[29], a decentralized autonomous power sharing control was firstly proposed for hybrid AC/DC microgrid by leveraging the advantages of both AC-frequency and DC-voltage deviations. In steady-state, it equalizes the
AC-side normalized frequency and DC-side normalized voltage, and determine the power reference for ILC. As a result, ILC functions as a 'virtual power wire', and the power can autonomously flow from under-loaded subgrid to overloaded subgrid. Then, a generalized $f_{a c}-P_{I L C}-V_{d c}$ threedimensional power control method of the ILC is proposed in [30] for standalone hybrid microgrid to reduce the control mode switching. In [31], a decentralized multi-time scale power control strategy is proposed to make the interlinked subgrids operate in coordination and support each other under the source/load power fluctuations. Besides, the decentralized control strategies for economic optimization have been expanded in [32]-[33] considering the global operation cost. On the whole, the aforementioned decentralized power control methods mainly focus on either global equal sharing operation [27]-[31] or decentralized economic operation [32]-[33]. They do not consider the diverse subgrids with different power priorities and capacities. When the power deficiency of microgrid clusters occurs due to the uncertainty of renewable generations, the power supply and power quality of critical subgrid with sensitive loads cannot be ensured. Therefore, the optimal coordination and support control among subgrids via ILCs are very crucial for the stable and resilient operation of the hybrid microgrid clusters.

To fill the gap of priority-driven decentralized operation, this study proposes a self-optimizing power control scheme of ILCs to guarantee the autonomous power exchange among microgrid clusters with different priorities and capacities. Compared with the communication-based methods [21]-[22], [25]-[26], the proposed optimal power control is achieved in a decentralized manner without any communication link. Thus, the proposed control algorithm has the features of decreased cost, increased scalability, reduced geographic restrictions and high resilience and robustness in terms of communication faults for industrial application. Moreover, compared with the existing decentralized control of ILCs [27]-[31], the core operation idea of interlinked-subgrid-coordination is progressed from "global-equal sharing" to "priority-driven optimizing". This study has two main works as below:

- Priority-driven optimal power exchange model of ILCs in decentralized manner. In [27]-[31], the autonomous operation mechanism of the ILC is inherently the "globalequal sharing" idea for only two microgrids. When the power shortage occurs, the power demand of critical microgrid with sensitive load cannot be guaranteed. Alternatively in this study, the flexible power flow principle for ILCs is built to supply the diverse subgrid demands. The optimal solution is indicated by fully considering the subgrid capacities and priorities. Specially, for a smallcapacity subgrid with critical load, its power supply and voltage quality is prioritized, such as in the applications of hospital, bank, police and data-center. Thus, the proposed method is based on the "priority-driven optimizing" idea.

- Optimal-oriented decentralized quasi-droop control of


an optimal-oriented quasi-droop control strategy of ILCs is developed in a decentralized manner. The voltage and 
frequency references of the ILC are obtained with local information. Compared with the current-controlled methods [27]-[31], an ancillary function of voltage regulation of ILCs is also provided in this study. When the AC voltage sources fail in AC subgrid, the ILC can provide a voltage regulation and the system has the fault-ride-through capability. TABLE I

Comparison of ILC Control Methods in Different Control Manners for Hybrid Microgrids

\begin{tabular}{|c|c|c|c|c|c|c|c|c|}
\hline \multirow{2}{*}{$\begin{array}{c}\text { Category } \\
\text { Comm. } \\
\text { dependency }\end{array}$} & \multirow[t]{2}{*}{$\begin{array}{c}\text { Refere } \\
\text { nce }\end{array}$} & \multicolumn{2}{|c|}{$\begin{array}{l}\text { 1) Optimal power } \\
\text { control }\end{array}$} & \multicolumn{4}{|c|}{ 2) Other functions } & \multirow{2}{*}{$\begin{array}{l}\text { 3) Comparison with existing methods } \\
\text { [21-22], [25-26] are communication-based methods. } \\
\text { This study and [27-31] are communication-less methods. }\end{array}$} \\
\hline & & $\begin{array}{l}\text { Global } \\
\text { equal } \\
\text { sharing }\end{array}$ & $\begin{array}{c}\text { Priority- } \\
\text { driven } \\
\text { optimizing }\end{array}$ & $\begin{array}{l}\text { Consider } \\
\text { subgrid } \\
\text { priority \& } \\
\text { capacity }\end{array}$ & $\begin{array}{l}\text { Accomm } \\
\text {-odate } \\
\text { multiple } \\
\text { subgrids }\end{array}$ & $\begin{array}{l}\text { DG power } \\
\text { uncertainty } \\
\text { complemen } \\
\text {-tarity }\end{array}$ & $\begin{array}{l}\text { Autonom } \\
\text {-ous load } \\
\text { shedding }\end{array}$ & \\
\hline $\begin{array}{l}\text { Centralized } \\
\text { comm. }\end{array}$ & [21-22] & & $\sqrt{ }$ & $\sqrt{ }$ & $\sqrt{ }$ & & $\sqrt{ }$ & \multirow{3}{*}{$\begin{array}{ll} & \text { Depending on high-bandwidth comm. networks; } \\
\times & \text { Comm. delay, packet-loss, and failure decreases } \\
& \text { system stability, reliability and resilience; } \\
\checkmark & \text { Optimal objectives are easily achieved. }\end{array}$} \\
\hline \multirow{2}{*}{$\begin{array}{l}\text { Distributed } \\
\text { comm. }\end{array}$} & [25] & $\sqrt{ }$ & & & & & & \\
\hline & [26] & & $\sqrt{ }$ & & $\sqrt{ }$ & & $\sqrt{ }$ & \\
\hline \multirow[b]{2}{*}{$\begin{array}{l}\text { Decentralized } \\
\text { without comm. }\end{array}$} & [27-31] & $\sqrt{ }$ & & & & & & $\begin{array}{ll}\mathbf{x} & \text { Without considering subgrid capacity and priority; } \\
\mathbf{x} & \text { For only two microgrids; } \\
\mathbf{x} & \text { Other functions are limited }\end{array}$ \\
\hline & $\begin{array}{l}\text { This } \\
\text { study }\end{array}$ & & $\sqrt{ }$ & $\sqrt{ }$ & $\sqrt{ }$ & $\sqrt{ }$ & $\sqrt{ }$ & $\begin{array}{ll}\checkmark & \text { Fully considering subgrid capacity and priority; } \\
\checkmark & \text { Decentralized manner without central comm.; } \\
\checkmark & \text { Improved resilience and robustness; reduced cost; } \\
\checkmark & \text { Subgrid plug-play and increased scalability. }\end{array}$ \\
\hline
\end{tabular}

To further highlight the contribution of the proposed decentralized self-optimizing power exchange control scheme, a comprehensive comparison with aforementioned control methods is presented in Table I, where control functions of ILC are discussed in different control manners. Comparatively, the proposed method has some merits: 1) self-optimizing power exchange control without communication; 2) ancillary service of quasi-droop-controlled voltage support for AC grid; 3) considering different subgrid priorities and capacities; 4) accommodating multiple subgrids; 5) features of uncertainty complementarity and autonomous load shedding.

The rest of this paper is organized as follows. In section II, the configuration of hybrid microgrid clusters and basic control are described. The proposed priority-driven selfoptimizing power control scheme is presented in section III. Control model and system stability are analyzed to adjust the parameters in section IV. In section V, three cases of HIL results are provided. Finally, the conclusion and future work follow in Section VI.

TABLE II

Operation Practice and Requisite of Different Real-world Microgrids ${ }^{[15],[34]-[35]}$

\begin{tabular}{|l|l|c|c|}
\hline $\begin{array}{c}\text { Load feature } \\
\text { within each } \\
\text { microgrid }\end{array}$ & \multicolumn{1}{|c|}{$\begin{array}{c}\text { Microgrid scenario } \\
\text { examples }\end{array}$} & $\begin{array}{c}\text { Voltage } \\
\text { deviation of } \\
\text { Power quality } \\
\text { requirement }\end{array}$ & $\begin{array}{c}\text { Sub-grid } \\
\text { priority } \\
\text { index }\end{array}$ \\
\hline $\begin{array}{l}\text { Class } \boldsymbol{A}: \\
\text { Critical load }\end{array}$ & $\begin{array}{l}\text { Hospital/bank/police/data } \\
\text { center microgrid }\end{array}$ & $\left|V / f_{\text {error }}\right|<0.5 \%$ & $\begin{array}{c}\text { High } \\
\mid \text { Cass }\end{array}$ \\
\hline $\begin{array}{l}\text { Class B: } \\
\begin{array}{l}\text { Fixed non- } \\
\text { critical load }\end{array}\end{array}$ & $\begin{array}{l}\text { Industrial/commercial } \\
\text { microgrid }\end{array}$ & $\left|V / f_{\text {error }}\right|<3 \%$ & $\begin{array}{c}\text { Medium } \\
\text { Class } \boldsymbol{C}:\end{array}$ \\
$\begin{array}{l}\text { Flexible non- } \\
\text { critical load }\end{array}$ & Residential midrogrid/ & $\left|V / f_{\text {error }}\right|<5 \%$ & Low \\
\hline
\end{tabular}

\section{Hybrid Microgrid CluSTERS ANd BASIC CONTROL}

\section{A. Configuration of Hybrid Microgrid Clusters}

Fig. 2 shows the typical configuration of hybrid microgrid clusters with four AC and DC microgrids. These subgrids are composed of various loads, dispatchable DGs, including storages, fuel cell, micro-turbine, etc., and non-dispatchable renewable generations. Four subgrids are interlinked by bidirectional ILCs, which includes DC/DC, DC/AC, and AC/AC ILCs for linking different microgrid types. Without loss of generality, this study focuses on the control technologies of DC/AC ILCs for hybrid AC/DC microgrid clusters.

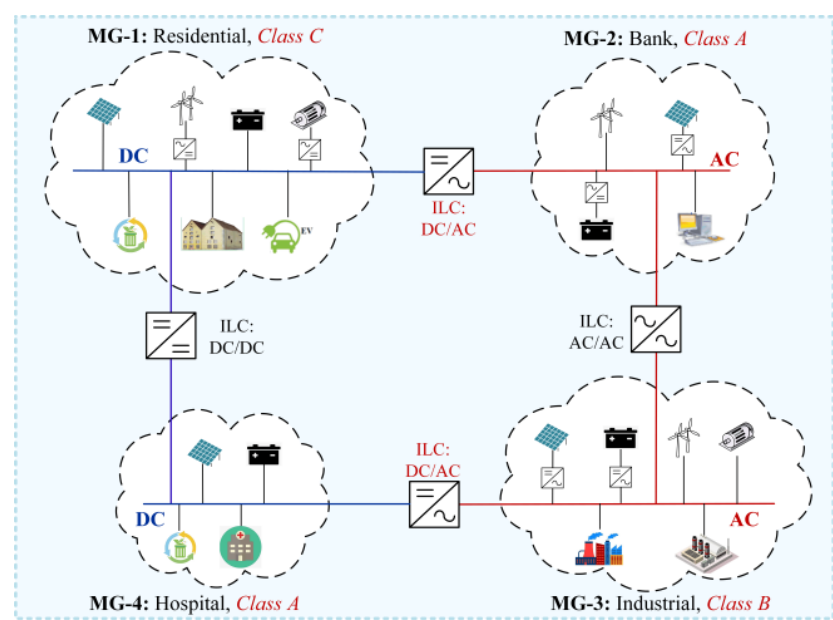

Fig. 2. A typical configuration of hybrid AC/DC microgrid clusters with different capacities and priorities.

Practically, in networked microgrid clusters, the subgrid load-priorities and power quality requirements for different areas can be different as shown in Table II. In our work, the microgrids are divided into three categories according to their individual fed-load features and priorities, namely Class $\boldsymbol{A}$ microgrid with critical load, Class $\boldsymbol{B}$-microgrid with fixed non-critical load, and Class $\boldsymbol{C}$-microgrid with flexible noncritical load [15], [34]-[35]. On this basis, the concept of subgrid priority index (important weight) is introduced to evaluate the importance of each subgrid, which lays a solid foundation of the optimal power exchange model in hybrid microgrid clusters. It is noted that the flexible load part of the low-priority subgrid could be curtailed or shifted according to the hierarchical segment of AC-frequency and DC-voltage deviations under the power shortage. 


\section{B. Desired Self-organizing Operation Mechanism of Hybrid Microgrid Clusters}

To control and manage the complex hybrid microgrid clusters, a self-organizing operation mechanism is necessary for a transition from a centralized system to a decentralized system [36]. In this study, a simple self-organizing operation rule is formed based on the proposed self-optimizing power flow principle. Some main features of self-organizing resilience operation are as follows:

$\diamond$ Decentralized self-optimizing manner. The decentralized self-optimizing power exchange control scheme of ILCs should be achieved by only local information, which is offline designed without requiring a central controller and has high system resilience to communication failure.

\& Reserved dispatchable source sharing. All dispatchable sources of microgrid clusters should be shared with each other according to the predesigned subgrid priorities.

s Mutual uncertainty complementarity. Due to the intermittency of wind/solar-based renewable generations and the load uncertainty in each subgrid, it is likely that the microgrid experiences power sufficiency and deficiency. To achieve mutual uncertainty complementarity, we should control the power flows of ILCs from the power-sufficiency subgrid to the power-deficiency subgrid.

$\checkmark$ Autonomous load shedding. The less-priority microgrid should shed load automatically by judging real-time frequency/voltage deviation when total power is in deficit.

$\diamond$ One-ILC-fault redundancy. The proposed control principle can accommodate one-ILC-failure.

\section{Basic Control of AC and DC Subgrids}

Many advanced control strategies for individual AC or DC microgrid have been maturely proposed in [41]-[43]. The normal operations of AC and DC subgrids mainly rely on parallel converters as well as the corresponding controller designs, which ensure the sufficient stability margin and dynamic responses. However, these damping controller designs just affect the dynamic operation without interfacing with the steady-state performance. For this paper, the focus is on the proposed priority-driven self-optimizing power control for ILCs in microgrid clusters, thus the compensation controller designs for AC and DC subgrids would not be discussed here in detail.

1) Control of non-dispatchable sources: To maximize the renewable energy harvest, power conversion control mode of renewable generations always works in maximum-powerpoint-tracking (MPPT), except for the extreme light-load conditions. Various MPPT control methods for different renewable power generations have been maturely developed. For the simplicity of demonstration, MPPT-controlled renewable power generations are considered as negative loads in this study.

2) Control for dispatchable sources: To establish AC and DC subgrid voltage and support MPPT-controlled renewable power generations, some dispatchable generators and energy storages are mostly needed to work in voltage control mode. Especially in islanded microgrid, one key challenge is the demand power sharing among dispatchable sources. To this issue, a classic droop control method has been proposed to realize decentralized power sharing objective based on the droop gains design [3]-[4]. Similarly, the decentralized droop control structure for proportional power sharing among all dispatchable resources is also applied in this study. To focus on the power exchange control scheme of ILCs for hybrid microgrid clusters, the droop-controlled dispatchable power sources in AC and DC subgrids are combined as one AC and one DC main voltage source for each subgrid.

In this section, classic droop control strategies for $\mathrm{AC}$ and DC subgrids are firstly introduced to regulate the power flow within each-subgrid separately. For AC subgrid, the droop control characteristic is shown in Fig. 3. The frequency reference $f_{a c}$ and the voltage amplitude reference $V_{a c}$ are presented as follows

$$
\begin{aligned}
& f_{a c}=f_{\max }-m P_{a c, \text { dispat }}, m=\frac{f_{\max }-f_{\min }}{P_{\mathrm{ac}, \max }-P_{\mathrm{ac}, \min }} \\
& V_{a c}=V_{a c, \text { max }}-n Q_{a c, \text { dispat }}, n=\frac{V_{\mathrm{ac}, \max }-V_{\mathrm{ac}, \min }}{Q_{\mathrm{ac}, \max }-Q_{\mathrm{ac}, \text { min }}}
\end{aligned}
$$

where $m$ and $n$ are the $P-f$ and $Q-V$ droop coefficients, respectively. $P_{a c, d i s p a t}$ and $Q_{a c, d i s p a t}$ are the output active and reactive power of dispatchable source in AC subgrid. From Fig. 3, $f_{\max }$ and $f_{\min }$ are the maximum and minimum values of permissible frequency. $V_{a c, \max }$ and $V_{a c, \min }$ are the maximum and minimum values of permissible voltage amplitude.



(a)

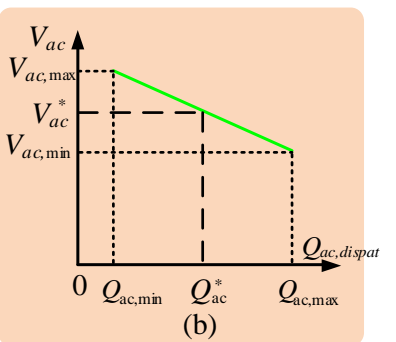

(b)
Fig. 3. Classic droop control for AC subgrid. (a) $P-f$ droop. (b) $Q-V$ droop.



Fig. 4. Classic $P-V$ droop control for DC subgrid.

As shown in Fig. 4, the $P-V$ droop control characteristic for DC subgrid is given by

$$
V_{d c}=V_{d c, \text { max }}-k P_{d c, \text { dispat }}, k=\frac{V_{\mathrm{dc}, \text { max }}-V_{\mathrm{dc}, \text { min }}}{P_{\mathrm{dc}, \text { max }}-P_{\mathrm{dc}, \text { min }}}
$$

where $P_{d c, \text { dispat }}$ is the output active power of dispatchable source in DC subgrid. $k$ is the $P-V$ droop coefficient. From Fig. $4, V_{d c, \max }$ and $V_{d c, \min }$ are the maximum and minimum values of permissible bus voltage amplitude. 


\section{Control Problem Description of ILCs}

According to the droop equations (1)-(3), the increase of load-demand within each-subgrid will lead to a decrease of the AC-subgrid frequency or DC-subgrid voltage. So, the relative deviations of AC-frequency and DC-voltage with respect to the rated-values are favorable indications of the real-time operation state-deviation in each-subgrid [27]-[29]. In this way, the normalized AC-frequency and DC-voltage are given by

$$
\left\{\begin{array}{l}
f_{p u}=\frac{f_{a c}-0.5\left(f_{\max }+f_{\min }\right)}{0.5\left(f_{\max }-f_{\min }\right)} \in[-1,1] \\
V_{d c, p u}=\frac{V_{d c}-0.5\left(V_{d c, \text { max }}+V_{d c, \text { min }}\right)}{0.5\left(V_{d c, \text { max }}-V_{d c, \text { min }}\right)} \in[-1,1]
\end{array}\right.
$$

Then, a uniform $\eta_{p u, i}$ of operation state deviation index for $i$-th AC or DC subgrid is given by

$$
\eta_{p u, i}= \begin{cases}f_{p u, i} ; & \text { For AC subgrid } \# i \\ V_{d c, p u, i} ; & \text { For DC subgrid \#i }\end{cases}
$$

Equation (5) is intended to unify expression forms of power sufficiency-deficiency states of AC-type and DC-type subgrid. For AC-type subgrid\# $i, \eta_{p u, i}=f_{p u, i}$. For DC-type subgrid\# $i, \quad \eta_{p u, i}=V_{d c, p u, i}$. This unified form can be used for a more convenient expression of the following optimal problem regardless of whether it's AC or DC subgrid. From (4)-(5), $\eta_{p u, i}=1$ represents power-sufficiency operation state; $\eta_{p u, i}=-$ 1 represents fully power-deficiency operation state; $\eta_{p u, i}=0$ represents nominal-load operation state of $i$-th subgrid.

The power exchange between microgrid clusters is coordinated by the ILC, as shown in Fig. 5. Different from the "global-equal sharing" concept in [27]-[29], this study focuses on two issues from the "priority-driven optimizing" perspective: 1) how to build the optimal power exchange model and manage the power flow among microgrid clusters by ILCs? 2) How to realize the control algorithm for ILCs in a decentralized manner to avoid the need of central controller and communication?

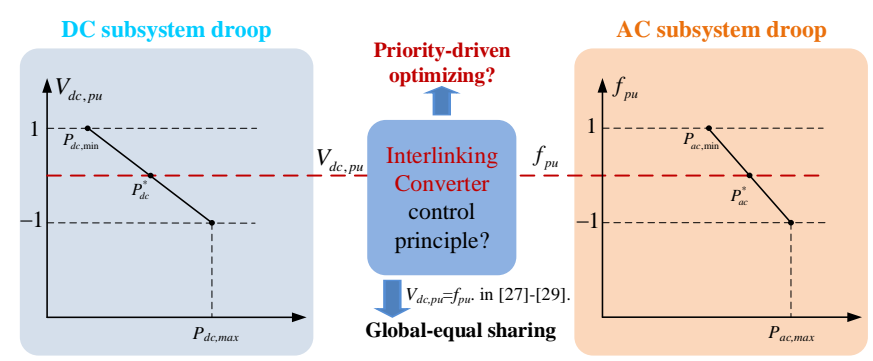

Fig. 5. Key control problem description of ILCs.

\section{Decentralized Self-optimizing Power EXCHANGE CONTROL SCHEME OF ILCS}

In this section, a priority-driven optimal power exchange model of ILCs is firstly introduced, and then optimal-oriented primary quasi-droop control of ILCs is implemented. The selfoptimizing power control scheme indicates the power exchange reference value for the primary control of the ILC. And the primary control is utilized to achieve the accurate tracking performances by dual-voltage-current closed loops.

\section{A. Flexible Priority-Driven Optimal Power Exchange Model} of ILCs Considering the Subgrid Priorities and Capacities

For this first issue, the question is how much power should be exchanged through ILC from the one subgrid to the other subgrid. Herein, a flexible priority-driven principle is introduced to address the optimal power exchange problem through ILCs. This optimal problem is summarized in (6).

In (6), $P_{I L C, i j}$ is the real power exchange of the ILC from the $i$-th subgrid to $j$-th subgrid. $w_{i}$ is the important weight coefficient of $i$-th subgrid priority. $\eta_{p u, i}$ is the deviation index of operation state for $i$-th subgrid, which implies the loadlevels of light-load or over-load. $N$ is the total subgrid number in hybrid microgrid clusters. $\Omega_{\mathrm{i}}$ represents the physically interlinked subgrids with the $i$-th subgrid. $P_{\text {load }, i}$ is the actual load demand of the $i$-th subgrid. $P_{\text {dispat } i}$ is output power of droop-controlled dispatchable sources in $i$-th subgrid. $P_{\text {dispat }, i}^{\max }$ is maximum power capacity of dispatchable sources in $i$-th subgrid. $P_{\text {non-dispat } i}$ is output power of MPPT-controlled nondispatchable sources in $i$-th subgrid, which can be treated as negative loads in the optimal model.

$$
\begin{aligned}
& \underset{P_{I L C, i j}}{\operatorname{minimize}} \quad J=\sum_{i=1}^{N} w_{i} \eta_{p u, i}^{2} \\
& \text { subject to } \\
& \left\{\begin{array}{l}
P_{\text {dispat }, i}-\sum_{j \in \Omega_{i}} P_{I L C, i j}=P_{\text {load }, i}-P_{\text {non-dispat }, i} \\
P_{\text {dispat }, j}+\sum_{i \in \Omega_{j}} P_{I L C, i j}=P_{\text {load }, j}-P_{\text {non-dispat }, j} \\
\sum_{i=1}^{N} P_{\text {dispat }, i}=\sum_{i=1}^{N}\left(P_{\text {load }, i}-P_{\text {non-dispat }, i}\right) \\
\eta_{p u, i}=1-\frac{2 P_{\text {dispat }, i}}{P_{\text {dispat }, i}^{\max }}
\end{array}\right.
\end{aligned}
$$

Equation (6) means the optimization objective by minimizing the total normalized DC-voltage/AC-frequency state deviations of microgrid clusters with different priorities. Equations (6a) and (6b) represent the supply-demand power balance of the $i$-th subgrid and $j$-th subgrid. Equations (6c) represent the supply-demand power balance of the whole $N$ microgrid clusters. Equation (6d) are deduced according to the normalization feature of droop-controlled subgrid from (1)-(5).

Then, a simplified mathematical description of optimal problem is obtained from (6)-(6d)

$$
\begin{aligned}
& \min \left(J=\sum_{i=1}^{N} w_{i} \eta_{p u, i}^{2}\right) \\
& \text { s.t. } \sum_{i=1}^{N} \frac{P_{d i s p a t, i}^{\max }\left(1-\eta_{p u, i}\right)}{2}=P_{\text {uncertainty }}
\end{aligned}
$$

where $P_{\text {uncertainty }}$ implies the equivalent load demands due to the uncertainty of renewable sources and load-feeding.

From (6)-(7), the optimization objective $J$ is the quadratic function of variable $\eta_{p u, i}$, and the second derivative Hessian Matrix $\quad \mathbf{H}(J)=\operatorname{diag}\left[\begin{array}{llll}2 w_{1} & 2 w_{2} & \cdots & 2 w_{N}\end{array}\right]$ is a positive definite matrix. So, the objective function $J$ is convex. Besides, the optimization constraint of (7) is a first-order function of 
variable $\eta_{p u, i}$, and the feasible region is a plane, which is convex. In brief, the optimization problem is convex, and a single minimum exists. Meanwhile, it is noted that this optimization problem focuses on the optimized power exchanges of ILCs from one subgrid to the other subgrid. The power loss of the line impedance and the node power equation within each subgrid are ignored.

Accordingly, the Lagrange function [37] is adopted to find the optimal solution in (7) as follow:

$$
L=\sum_{i=1}^{N} w_{i} \eta_{p u, i}^{2}+\lambda\left(P_{\text {uncertainty }}-\sum_{i=1}^{N} \frac{P_{d i s p a t, i}^{\max }\left(1-\eta_{p u, i}\right)}{2}\right)
$$

where $\lambda$ is Lagrange multiplier. Therefore, the optimum solution is obtained by solving the following equation:

$$
\left\{\begin{array}{l}
\frac{\partial L}{\partial \eta_{p u, 1}}=0, \frac{\partial L}{\partial \eta_{p u, 2}}=0, \ldots, \frac{\partial L}{\partial \eta_{p u, N}}=0 \\
\frac{\partial L}{\partial \lambda}=P_{\text {uncertainty }}-\sum_{i=1}^{N} \frac{P_{d i s p a t, i}^{\max }\left(1-\eta_{p u, i}\right)}{2}=0
\end{array}\right.
$$

Simplifying (9) yields the necessary condition (10) for the optimality.

$$
\frac{w_{1}}{P_{\text {dispat }, 1}^{\max }} \eta_{p u, 1}=\frac{w_{2}}{P_{\text {dispat }, 2}^{\max }} \eta_{p u, 2}=\cdots=\frac{w_{N}}{P_{\text {dispat }, N}^{\max }} \eta_{p u, N}
$$

From (10), although the value of $P_{I L C, i j}$ is difficult to be directly solved due to the load uncertainty, it can be easily obtained that the minimum of objective function (6) can be achieved autonomously when the condition (10) is met. As the microgrid clusters are physically connected by all ILCs, (10) can be held by controlling each ILC in a decentralized manner. Moreover, the steady-state operation deviation index $\eta_{p u, i}$ is a constant value. Thus, the desired power reference for each ILC can be designed by a proportional-integral (PI) controller with steady-state zero-error to guarantee (10) hold.

$$
P_{I L C, i j}^{*}=\left(k_{p}+\frac{k_{i}}{s}\right)\left(\frac{w_{i}}{P_{\text {dispat }, i}^{\max }} \eta_{p u, i}-\frac{w_{j}}{P_{\text {dispat }, j}^{\max }} \eta_{p u, j}\right)
$$

where $P_{I L C, i j}^{*}$ implies the optimized power exchange value of the $I L C_{i j}$ from the $i$-th subgrid to $j$-th subgrid.

From (10)-(11), it is noted that the priority weight $w_{i}$ and the maximum dispatchable power capacity $P_{\text {dispat }, i}^{\max }$ of the $i$-th subgrid can be calculated off-line according to their individual subgrid priority and capacity. Hereby, the proposed power flow principle is flexible and can be designed according to the application scenarios of hybrid microgrid clusters.

Practically, the concept of subgrids' priority weights are introduced to evaluate the load importance classes of each subgrid, which can be defined as the proportion of important customers to total customers in a certain subgrid [38]-[39]. And these weight values are normally designed according to the voltage deviation criteria of power quality requirement for different subgrids, as shown in Table II. For a better understanding, a simple design procedure is illustrated in Fig. 6. For simplicity, we assume three subgrids with same power capacities. From the optimum solution of (10), the steady-state equation $w_{1} \eta_{p u, 1}=w_{2} \eta_{p u, 2}=w_{3} \eta_{p u, 3}$ must be firstly guaranteed. Then, according to the voltage deviation requirement of power quality in Table II, we set the high-priority subgrid-1 with $\left|V / f_{\text {error }, 1}\right|<0.5 \%$, the medium-priority subgrid-2 with $\left|V / f_{\text {error }, 2}\right|$ $<3 \%$, and the low-priority subgrid-3 with $\left|V / f_{\text {error }, 2}\right|<5 \%$. As a result, $\eta_{p u, 1}: \eta_{p u, 2}: \eta_{p u, 3}=|V| f_{\text {error }, 1}|:| V\left|f_{\text {error }, 2}\right|:|V| f_{\text {error }, 3} \mid=0.5: 3: 5$. And $w_{1}: w_{2}: w_{3}=10: 1.66: 1$ is derived.

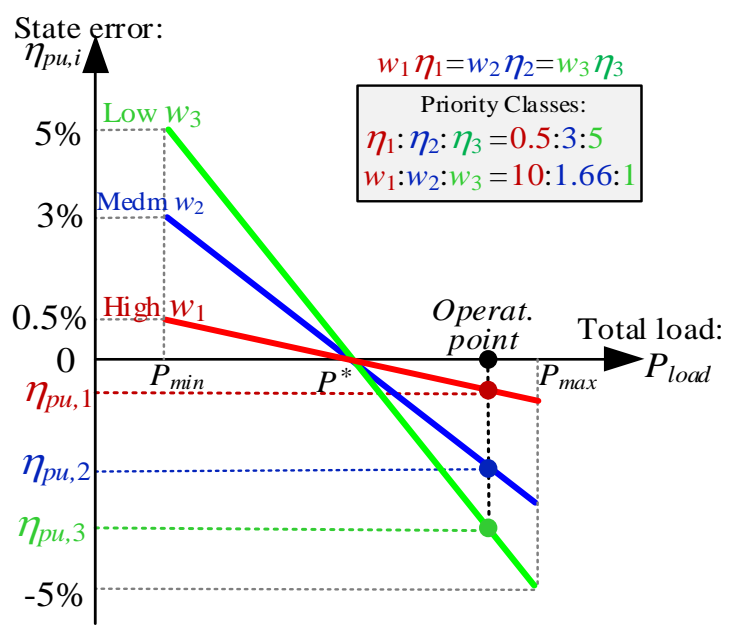

Fig. 6. Simplified design diagram of subgrids with different priority weights.

From Fig. 6, a higher weight $w_{i}$ implies a higher priority of power demand and higher voltage quality. Specially, the power supply and voltage quality of the high-priority subgrid1 would be guaranteed while the low-priority subgrid-3 can be compromised. Under the power over-shortage, the voltage/frequency state deviation of the low-priority subgrid-3 is relatively large, and the flexible load part of the subgrid-3 could be curtailed or shifted by under-frequency/voltage loadshedding. It is noted that the autonomous load shedding is carried out by judging local frequency/voltage deviation within each subgrid, and the deviations are not shared among subgrids. For overall microgrid clusters, the autonomous load shedding can be equivalent to cutting off part of the load to guarantee the priority-driven power supply, which does not affect the effectiveness of our proposed method.

\section{B. Optimal-Oriented Decentralized Quasi-Droop Control Realization of ILCs}

To achieve the control objective in (10)-(11), a quasidroop control is proposed for the $I L C_{i j}$.

$$
\begin{gathered}
f_{I L C, i j}=f_{I L C}^{*}+\left(k_{I L C p, i j}+\frac{k_{I L C i, i j}}{s}\right)\left(P_{I L C, i j}^{*}-P_{I L C, i j}\right) \\
V_{I L C, i j}=V_{I L C, \max }-n_{I L C, i j} Q_{I L C, i j}
\end{gathered}
$$

where $k_{I L C p, i j}$ and $k_{I L C i, i j}$ are the PI coefficients of $P$ - $f$ quasidroop, respectively. $n_{I L C, i j}$ is the $Q-V$ quasi-droop coefficient. $P_{I L C, i j}$ is the active power exchange of the ILC from the $i$-th subgrid to $j$-th subgrid, and $P_{I L C, i j}=-P_{I L C, j i}$. The power flow direction of a positive active power is assumed from $i$-th DC side to $j$-th $\mathrm{AC}$ side. When the power value is more than zero, the ILC works in inverter mode, and when less than zero, the ILC works in rectifier mode. $Q_{I L C, i j}$ is the output reactive power of the ILC from the $i$-th DC subgrid to $j$-th AC subgrid. 
$V_{I L C, i j}$ is the voltage amplitude reference of the $I L C_{i j}$. In (12), the frequency reference is generated according to the real-time operation states of interlinked AC and DC subgrids. Due to the steady state zero-error of PI controller, $P_{I L C, i j}^{*}=P_{I L C, i j}$ and $\frac{w_{i}}{P_{d i s p a t, i}^{\max }} \eta_{p u, i}=\frac{w_{j}}{P_{d i s p a t, j}^{\max }} \eta_{p u, j}$ in steady-state, and the above optimal principle (6)-(10) is achieved autonomously. In (13), the $Q-V$ droop control is adopted for the voltage control and reactive power compensation to AC subgrid. The detailed voltagebased control scheme for the $I L C_{i j}$ is shown in Fig. 7. The proposed control (12)-(13) is used to indicate the inner voltage reference for dual-voltage-current closed loops. The voltage and current references are tracked by the proportional-resonant (PR) control.

Moreover, it is noted that the interlinking converters can operate as a voltage-controlled-source or current-controlled source. The control mode is always chosen according to the practical requirements of current dynamic response, power quality and the stability of grid strengths [44]. Comparatively, the voltage-controlled mode for ILCs can work in the weak grid condition, and provide an ancillary function of voltage regulation.

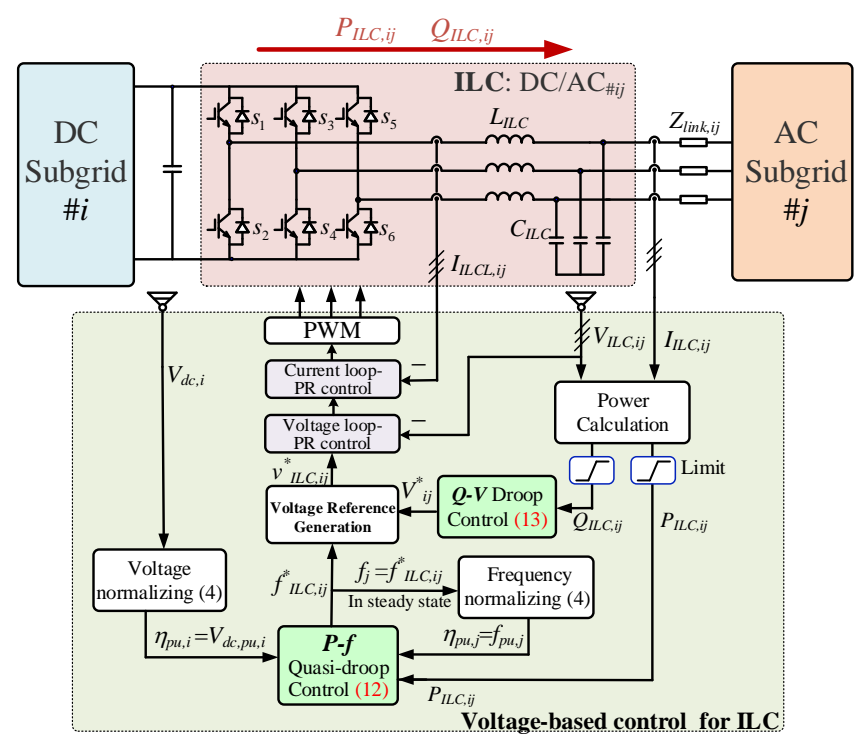

Fig. 7. Proposed voltage-based quasi-droop control for $I L C_{i j}$.

\section{Stability AnALYSIS OF ILCS}

Stability analysis and controller designs are essential for the normal operation of the interlinking converters. Thus the small signal stability analysis for the ILCs is carried out in this section. Several basic assumptions are highlighted before modeling the ILCs. i) The dynamics of dual-voltage-current closed control loops (hundreds of hertz) are overlooked, considering the timescale of interest in this study is focused on the power exchange control dynamics (typically $2 \sim 15$ hertz). ii) The resistive component and electromagnetic transients in the line circuit are neglected. iii) The voltage amplitude of the ILC can be treated as a constant due to the salient time-scale separation between the dynamics of frequency control and voltage control. iv) The DC-link and AC-link bus dynamics of the ILC are assumed to the droop controlled features of (1), (3) with feeding constant power loads.

\section{A. Overall Control Model of the ILC}

First, the power transmission model of the ILC is built.

$$
P_{I L C}=\frac{V_{I L C} V_{a c}}{Z_{\text {link }}} \sin \left(\delta_{I L C}-\delta_{a c}\right)
$$

where $V_{I L C}$ and $\delta_{I L C}$ are the voltage magnitude and angle of the ILC, respectively. $V_{a c}$ and $\delta_{a c}$ are the voltage magnitude and angle of the AC-link connection bus, respectively. $Z_{\text {link }}$ is the line reactance between the ILC and AC-link bus.

Then, linearizing (1), (3)-(6), (11)-(12) and (14) around the steady state points yields

$$
\left\{\begin{array}{l}
\dot{\tilde{\delta}}_{a c}=\tilde{f}_{a c}=-m \tilde{P}_{a c, d i s p a t} ; \quad \tilde{V}_{d c}=-k \tilde{P}_{d c, d i s p a t} \\
\tilde{\eta}_{p u, a c}=\tilde{f}_{p u}=a_{f} \tilde{f}_{a c} ; \quad \tilde{\eta}_{p u, d c}=\tilde{V}_{d c, p u}=a_{V} \tilde{V}_{d c} \\
\tilde{P}_{I L C}^{*}=k_{p}\left(w_{d c p} \tilde{\eta}_{p u, d c}-w_{a c p} \tilde{\eta}_{p u, a c}\right)+\tilde{\Phi}_{I L C} \\
\dot{\tilde{\Phi}}_{I L C}=k_{i}\left(w_{d c p} \tilde{\eta}_{p u, d c}-w_{a c p} \tilde{\eta}_{p u, a c}\right) \\
\dot{\tilde{\delta}}_{I L C}=\tilde{f}_{I L C}=k_{I L C p}\left(\tilde{P}_{I L C}^{*}-\tilde{P}_{I L C}\right)+\tilde{\Theta}_{I L C} \\
\dot{\tilde{\Theta}}_{I L C}=k_{I L C i}\left(\tilde{P}_{I L C}^{*}-\tilde{P}_{I L C}\right) \\
\tilde{P}_{I L C}=P_{I L C}^{\max }\left(\tilde{\delta}_{I L C}-\tilde{\delta}_{a c}\right) ; \\
\tilde{P}_{d c, \text { dispat }}-\tilde{P}_{I L C}=\tilde{P}_{d c, \text { load }}=0 ; \quad \tilde{P}_{a c, \text { dispat }}+\tilde{P}_{I L C}=\tilde{P}_{a c, \text { load }}=0
\end{array}\right.
$$

where ‘ $\sim$ denotes small perturbation around equilibrium points. And

$$
\left\{\begin{array}{l}
a_{f}=\frac{1}{0.5\left(f_{\max }-f_{\min }\right)} ; a_{V}=\frac{1}{0.5\left(V_{d c, \max }-V_{d c, \min }\right)} \\
w_{\text {acp }}=\frac{w_{i}}{P_{\text {dispat }, a c}^{\max }} ; w_{d c p}=\frac{w_{i}}{P_{d i s p a t, d c}^{\max }} \\
P_{I L C}^{\max }=\frac{V_{I L C} V_{a c}}{Z_{\text {link }}} \cos \left(\delta_{I L C}-\delta_{a c}\right)
\end{array}\right.
$$

Rewriting (15) in matrix form, the state-space equations of the power control stability of the ILC is presented as

$$
\left[\begin{array}{c}
\dot{\tilde{\delta}}_{a c} \\
\dot{\tilde{\Phi}}_{I L C} \\
\dot{\tilde{\delta}}_{I L C} \\
\dot{\tilde{\Theta}}_{I L C}
\end{array}\right]=\left[A_{I L C}\right]\left[\begin{array}{c}
\tilde{\delta}_{a c} \\
\tilde{\Phi}_{I L C} \\
\tilde{\delta}_{I L C} \\
\tilde{\Theta}_{I L C}
\end{array}\right]
$$

where $a_{I L C}=P_{I L C}^{\max }\left(w_{d c p} a_{V} k+w_{a c p} a_{f} m\right)$.

$$
\left[A_{I L C}\right]=\left[\begin{array}{cccc}
-m P_{I L C}^{\max } & 0 & m P_{I L C}^{\max } & 0 \\
k_{i} a_{I L C} & 0 & -k_{i} a_{I L C} & 0 \\
k_{I L C p} P_{I L C}^{\max }+k_{I L C p} k_{p} a_{I L C} & k_{I L C p} & -k_{I L C p} P_{I L C}^{\max }-k_{I L C p} k_{p} a_{I L C} & 1 \\
k_{I L C i} P_{I L C}^{\max }+k_{I L C i} k_{p} a_{I L C} & k_{I L C i} & -k_{I L C i} P_{I L C}^{\max }-k_{I L C} k_{p} a_{I L C} & 0
\end{array}\right]
$$

\section{B. Eigenvalue Analysis}

The eigenvalues of matrix $\mathrm{A}_{\mathrm{ILC}}$ in (18) can be used to study the stability of the ILC around the state of equilibrium. For the HIL test system to be described later in Section V, the root-locus plots of matrix $\mathrm{A}_{\mathrm{ILC}}$ for the ILC\#12 are shown in Fig. 8 while varying the control parameters, $k_{I L C p}$ and $k_{I L C i}$. 
Fig. 8(a) shows the root locus with the proportional parameter $k_{I L C p}$ changing from $1 \mathrm{e}^{-5}$ to $1 \mathrm{e}^{-3}$. In Fig. 8(a), there is a simple eigenvalue at zero corresponding to rotational symmetry, which has been proven in [40]. And the rest eigenvalues are in the left half-plane when $k_{I L C p}>3 \mathrm{e}^{-5}$. With $k_{I L C p}$ increases in Fig. 8(a), the dominant eigenvalues $\left(\lambda_{1}\right.$ and $\left.\lambda_{2}\right)$ gradually move away from the imaginary axis. Meanwhile, the eigenvalue $\lambda_{3}$ moves close to the imaginary axis, which makes the system more easy to become unstable. Therefore, it is important to choose a suitable value of the proportional parameter $k_{I L C p}$ to ensure the stability of the ILC. Fig. 8(b) shows the root locus as the integral parameter $k_{I L C i}$ increases from $1 \mathrm{e}^{-5}$ to $3 \mathrm{e}^{-3}$. In this case, there also exists an eigenvalue at zero [40]. When the value of $k_{I L C i}$ becomes large, the dominant pole $\lambda_{1}$ moves away from the imaginary axis. And $\lambda_{2}$ and $\lambda_{3}$ gradually move away from the real axis, which decreases the damping ratio of the system. Thus, it is important to choose a suitable value of the integral parameter $k_{I L C i}$ to ensure the satisfactory dynamic response of the system.



(a)



(b)

Fig. 8. Eigenvalues of state-space matrix $\mathrm{A}_{\text {ILC }}$ for the ILC\#12. (a) $1 \mathrm{e}^{-5}<k_{I L C p}$ $<1 \mathrm{e}^{-3}$, (b) $1 \mathrm{e}^{-5}<k_{I L C i}<3 \mathrm{e}^{-3}$.

\section{HARDWARE-IN-LOOP (HIL) TESTS}

The proposed priority-driven self-optimizing power flow control scheme of ILCs is verified by real-time HIL tests. As shown in Fig. 9, the HIL system includes two parts: physical main circuits and algorithm controller. The physical circuits are realized by the real-time simulator OP5600 with $20 \mu \mathrm{s}$ time-step, which can accurately and effectively mimic the dynamics of the power electronic components. The algorithm controller is the real-hardware dSPACE1202 Microlab-box, and the sampling frequency is $20 \mathrm{kHz}$.

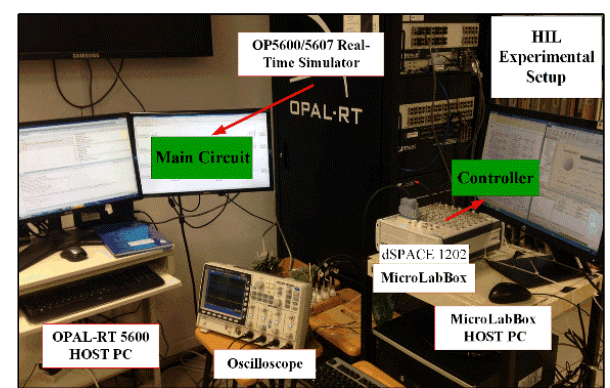

(a) Setup of the HIL test platform. Main circuit part realized by real-time simulator



(b) Diagram of the HIL test platform.

Fig. 9. Hardware-in-loop (HIL) test platform.

TABLE III

HIL System Parameters

\begin{tabular}{|c|c|c|}
\hline Description & Symbol & Value \\
\hline \multicolumn{3}{|c|}{ Physical parameters of four subgrids } \\
\hline $\begin{array}{l}\text { DC subgrid voltage } \\
\text { AC subgrid frequency } \\
\text { AC subgrid voltage } \\
\text { Priority weight } \\
\text { Max. dispat. power cap. } \\
\text { Nom. dispat. power cap. } \\
\text { Nom. load active power } \\
\text { Nom. load reactive power }\end{array}$ &  & $\begin{array}{c}700 / 685 / 670 \mathrm{~V} \\
50.5 / 50 / 49.5 \mathrm{~Hz} \\
321 / 311 / 301 \mathrm{~V} \\
\mathbf{3} / \mathbf{1} / \mathbf{1} / \mathbf{1} \\
\mathbf{5} / \mathbf{5} / \mathbf{1 0} / \mathbf{1 0} \mathbf{k W} \\
2.5 / 2.5 / 5 / 5 \mathrm{~kW} \\
2.5 / 2.5 / 5 / 5 \mathrm{~kW} \\
0 / 2 / 0 / 3 \mathrm{kVar} \\
\end{array}$ \\
\hline \multicolumn{3}{|c|}{ Control parameters of ILCs } \\
\hline $\begin{array}{l}\text { Ref. voltage/ frequency } \\
Q-V \text { droop coefficient } \\
P-f \text { droop PI coefficient } \\
\text { Power control Coefficient }\end{array}$ & $\begin{array}{c}V_{I L C, \max } / f^{*}{ }_{L L C} \\
n_{I L C} \\
k_{I L C p} / k_{I L C i} \\
k_{p} / k_{i}\end{array}$ & $\begin{array}{c}321 \mathrm{~V} / 50 \mathrm{~Hz} \\
3 \mathrm{e}^{-3} \\
2 \mathrm{e}^{-4} / 1 \mathrm{e}^{-4} \\
5 \mathrm{e}^{2} / 1 \mathrm{e}^{4}\end{array}$ \\
\hline
\end{tabular}



Fig. 10. Studied system model of four hybrid AC/DC microgrids.

The studied system model of four hybrid AC/DC microgrids is described in Fig. 10. Subgrid-1 and subgrid-3 are DC type. Subgrid-2 and subgrid-4 are AC type. There are four H-bridge-based bidirectional ILCs to interlink the four subgrids, ILC\#12, ILC\#32, ILC\#34 and ILC\#14. The proposed decentralized quasi-droop control scheme of four ILCs is shown in Fig. 8. The power flow direction reference of 
a positive active power is assumed from DC side to AC side. For example, $P_{I L C, 12}$ implies the power exchange value of ILC\#12 from subgrid-1 to subgrid-2. When the power value is more than zero, the ILC works in inverter mode, and when less than zero, the ILC works in rectifier mode.

The physical and control parameters in HIL system are listed in Table III. The priority weights $w_{i}$ of four subgrids are $3,1,1$, and 1, respectively. The DC subgrid-1 has a high priority of power supply and high requirement of voltage deviation. Besides, the dispacthable power capacity of subgrid-3/subgrid-4 is twice as much as that of subgrid-1/ subgrid-2. The nominal power capacities of four subgrids are $2.5,2.5,5$, and $5 \mathrm{~kW}$, respectively. From the optimal solution in (10), the following theoretical condition should be enabled in test results to minimize the optimization objection (6).

$$
\begin{cases}\frac{\eta_{p u, 1}}{1}=\frac{\eta_{p u, 2}}{3}=\frac{\eta_{p u, 3}}{6}=\frac{\eta_{p u, 4}}{6} ; & \text { This study } \\ \frac{\eta_{p u, 1}}{1}=\frac{\eta_{p u, 2}}{1}=\frac{\eta_{p u, 3}}{1}=\frac{\eta_{p u, 4}}{1} ; & \text { in [27]-[29] }\end{cases}
$$

To verify the priority-driven optimizing result of this study, a comparison with existing "global equal sharing" method [27]-[29] is carried out in Case I. Then, to further verify the redundancy of single point of ILC failure, Case II is built under ILC\#14 failure. To analysis the impact of a large difference between priority weights on the transient process, Case III with $w_{1}=5$ is tested under the load-change condition.

\section{A. Case I: Proposed Priority-driven Self-optimizing Power Control and Its Comparison with Existing Global Equal-} sharing Method [27]-[29]

Fig. 11 shows the test waveforms of Case I. The detailed steady-state values are concluded and analyzed in Table IV. The load of subgrid- 4 increases from $2 \mathrm{~kW}$ to $8 \mathrm{~kW}$ at $\mathrm{t}=4 \mathrm{~s}$. The load of the other three subgrids are in nominal values, whose values are $2.5 \mathrm{~kW}, 2.5 \mathrm{~kW}$, and $5 \mathrm{~kW}$, respectively. Before $4 \mathrm{~s}$, the total nominal dispatchable power capacity of four subgrids is $15 \mathrm{~kW}$, and actual active power of total load demand is $12 \mathrm{~kW}$. Thus, the total active power is surplus (in power-sufficiency), and the operation state deviation indexes of four subgrids are positive in Fig. 11 (f.1)-(f.2). After 4s, the total load power becomes $18 \mathrm{~kW}$, which is more than $15 \mathrm{~kW}$ at a power deficit state (in power-dificiency), and then the operation state deviation indexes of four subgrids becomes negative in Fig. 11 (f.1)-(f.2). From Table IV, the steady-state deviation indexes $\eta_{p u, i}$ of four subgrids are $0.04 / 0.12 / 0.24 /$ 0.24 at $\mathrm{t} \in[0,4 \mathrm{~s}]$ and $-0.04 /-0.12 /-0.24 /-0.24$ at $\mathrm{t} \in[4 \mathrm{~s}, 10 \mathrm{~s}]$ under the proposed method. Clearly, two different operation states are opposite and symmetrical based on the nominal operation state. And thus, they have the same optimized object. In the existing global equal-sharing method [27]-[29], Fig. 11 (f.2) and Table IV show that the steady-state deviation indexes $\eta_{p u, i}$ of four subgrids are $0.2 / 0.2 / 0.2 / 0.2$ at $\mathrm{t} \in[0,4 \mathrm{~s}]$ and $-0.2 /-0.2 /-0.2 /-0.2$ at $t \in[4 \mathrm{~s}, 10 \mathrm{~s}]$. All indexes of four subgrids are always same because of the same priority weight before and after $4 \mathrm{~s}$.
From Table IV and Fig. 11 (f.1)-(f.2), some connotations about state indexes are drawn under the proposed method:

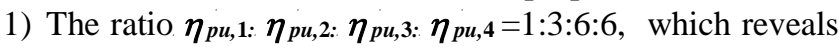
that equation (19) always holds, which verify the effectiveness of the above theoretical optimal mode and proposed control scheme.

2) Compared with the global equal-sharing method, we can see that, subgrid-1 with high priority weight has a highest voltage quality index and a high priority of power demand, which verifies the proposed priority-driven optimizing concept.

3) Subgrid-3 and subgrid-4 with same capacity and priority have a same state deviation index.

4) Subgrid-2 with small capacity has a better state deviation index than subgrid-3 and subgrid-4 because the power demand of a small-capacity subgrid can be easily met.

5) When total power is heavily deficit, subgrid-3 and subgrid-4 will shed load automatically by judging the large state deviations in order to guarantee the total power balance of high-priority subgrid.

6) The optimized objective value of (6) under the proposed method is $J=0.1344$, which is better than the existing method $J=0.24$.

$$
\left\{\begin{array}{l}
P_{d c, 1}-P_{I L C, 12}-P_{I L C, 14}=P_{\text {load }, 1} \\
P_{a c, 2}+P_{I L C, 12}+P_{I L C, 32}=P_{\text {load }, 2} \\
P_{d c, 3}-P_{I L C, 32}-P_{I L C, 34}=P_{\text {load }, 3} \\
P_{a c, 4}+P_{I L C, 34}+P_{I L C, 14}=P_{\text {load }, 4} \\
P_{d c, 1}+P_{a c, 2}+P_{d c, 3}+P_{a c, 4}=P_{\text {load }, 1}+P_{\text {load }, 2}+P_{\text {load }, 3}+P_{\text {load }, 4}
\end{array}\right.
$$

\begin{tabular}{|c|c|c|c|c|c|}
\hline \multirow[b]{2}{*}{$\begin{array}{l}\text { Steady- } \\
\text { state } \\
\text { analysis }\end{array}$} & \multirow[b]{2}{*}{$\begin{array}{c}\text { Power } \\
(\mathrm{kW})\end{array}$} & \multicolumn{2}{|c|}{ Proposed method } & \multicolumn{2}{|c|}{ Existing method } \\
\hline & & $\begin{array}{c}\mathbf{t} \in[\mathbf{0}, \mathbf{4 s}] \\
\text { power } \\
\text { surplus }\end{array}$ & $\begin{array}{c}\mathbf{t} \in[\mathbf{4 s}, \mathbf{1 0 s}] \\
\text { power } \\
\text { deficit } \\
\end{array}$ & $\begin{array}{c}\mathbf{t} \in[\mathbf{0}, \mathbf{4 s}] \\
\text { power } \\
\text { surplus }\end{array}$ & $\begin{array}{c}\mathbf{t} \in[\mathbf{4 s}, \mathbf{1 0 s}] \\
\text { power } \\
\text { deficit }\end{array}$ \\
\hline \multirow{2}{*}{ Subgrid-1 } & $P_{d c, 1}$ & 2.4 & 2.6 & 2.0 & 3.0 \\
\hline & $P_{l o a d, 1}$ & \multicolumn{2}{|c|}{2.5} & \multicolumn{2}{|c|}{2.5} \\
\hline ILC \#1-2 & $P_{I L C, 12}$ & 0.2 & -0.2 & 0.35 & -0.35 \\
\hline \multirow{2}{*}{ Subgrid-2 } & $P_{a c, 2}$ & 2.2 & 2.8 & 2.0 & 3.0 \\
\hline & $P_{\text {load }, 2}$ & \multicolumn{2}{|c|}{2.5} & \multicolumn{2}{|c|}{2.5} \\
\hline ILC \#3-2 & $P_{I L C, 32}$ & 0.1 & -0.1 & 0.15 & -0.15 \\
\hline \multirow{2}{*}{ Subgrid-3 } & $P_{d c, 3}$ & 3.8 & 6.3 & 4.0 & 6.0 \\
\hline & $P_{\text {load }, 3}$ & \multicolumn{2}{|c|}{5} & \multicolumn{2}{|c|}{5} \\
\hline ILC \#3-4 & $P_{I L C, 34}$ & -1.3 & 1.4 & -1.15 & 1.15 \\
\hline \multirow{2}{*}{ Subgrid-4 } & $P_{a c, 4}$ & 3.6 & 6.3 & 4.0 & 6.0 \\
\hline & $P_{l o a d, 4}$ & 2 & 8 & 2 & 8 \\
\hline ILC \#1-4 & $P_{I L C, 14}$ & -0.3 & 0.3 & -0.85 & 0.85 \\
\hline $\begin{array}{c}\text { Operation } \\
\text { state } \\
\text { deviations }\end{array}$ & $\begin{array}{l}\eta_{p u, 1--} \\
\eta_{p u, 4}\end{array}$ & $\begin{array}{c}0.04 / 0.12 / \\
0.24 / 0.24\end{array}$ & $\begin{array}{c}-0.04 /-0.12 / \\
-0.24 /-024\end{array}$ & $\begin{array}{c}0.2 / 0.2 / \\
0.2 / 0.2\end{array}$ & $\begin{array}{l}-0.2 /-0.2 / \\
-0.2 /-0.2\end{array}$ \\
\hline $\begin{array}{l}\text { Optimized } \\
\text { Object }(6)\end{array}$ & $J$ & 0.1344 & 0.1344 & 0.24 & 0.24 \\
\hline
\end{tabular}

TABLE IV

Comparative Analysis of Proposed Method with Existing Method [27]-[29]

Moreover, for the power exchanges among four subgrids in Fig. 11 (a.1)-(b.1), Table IV presents the detailed data, and the power flow chart is also briefly given in Fig.12. At $t \in[0$, $4 \mathrm{~s}$, subgrid-4 is under power sufficiency, then the dispatchable active power in subgrid-4 is transferred by ILCs to the other three subgrids. ILC\#34 and ILC\#14 work in the rectifier mode, and ILC\#12 works in the inverter mode. At $\mathrm{t} \in[4 \mathrm{~s}, 10 \mathrm{~s}]$, subgrid-4 is under power deficiency, then the dispatchable active power is transferred by ILCs from other 


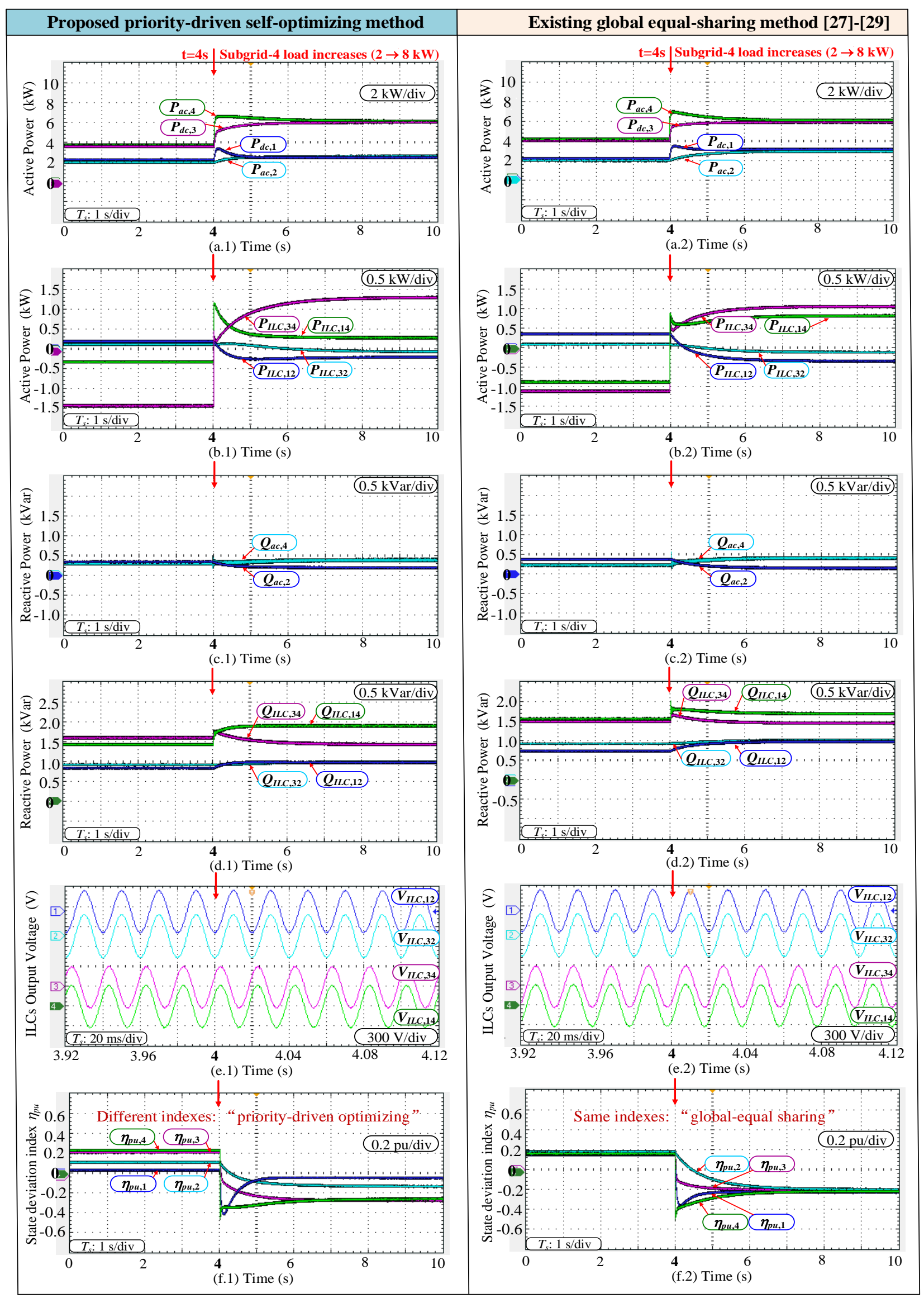

Fig. 11. Comparisons of the proposed priority-driven self-optimizing method with the existing global equal-sharing method [27]-[29]. (a) Output active power of four subgrids, (b) active power of four ILCs, (c) reactive power of two AC subgrids, (d) reactive power of four ILCs, (e) output voltage of four ILCs, and (f) operation state deviation index of four subgrids. 


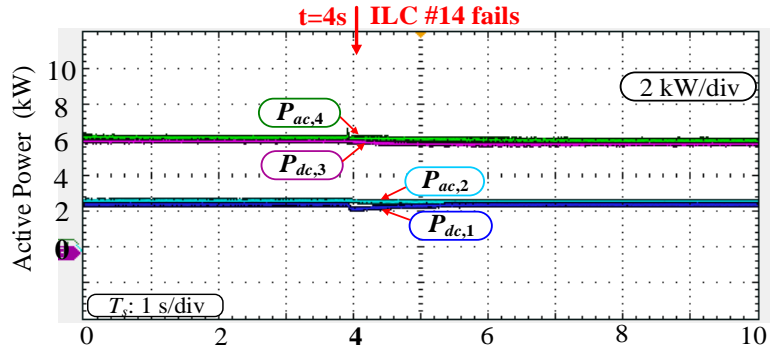

(a) Time (s)

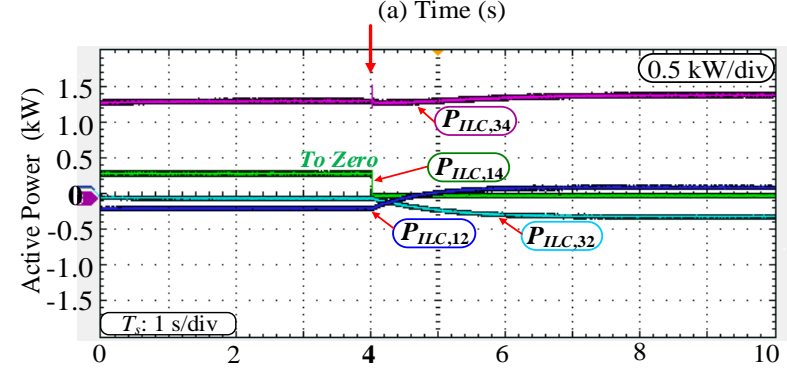

(b) Time (s)

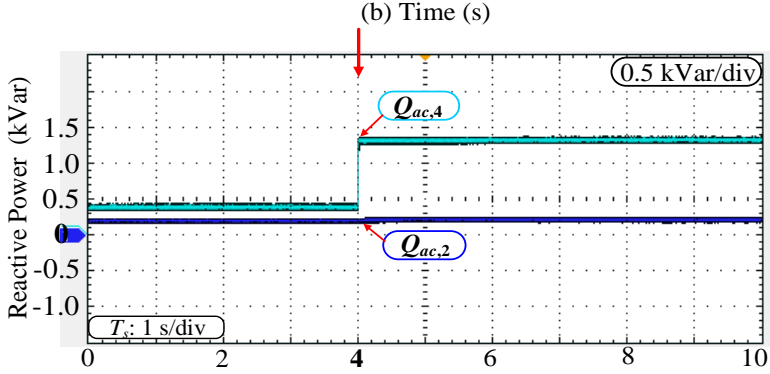

(c) Time (s)

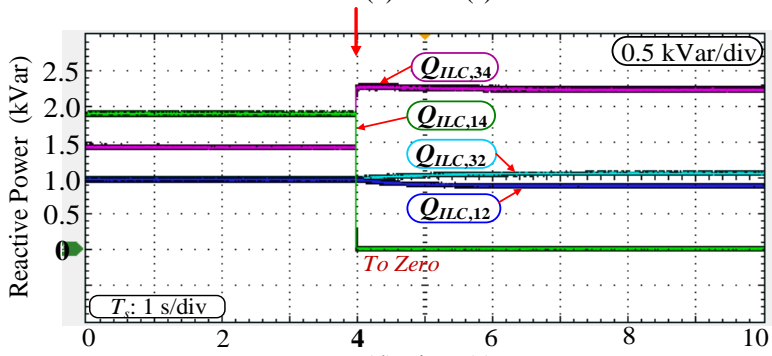

(d) Time (s)

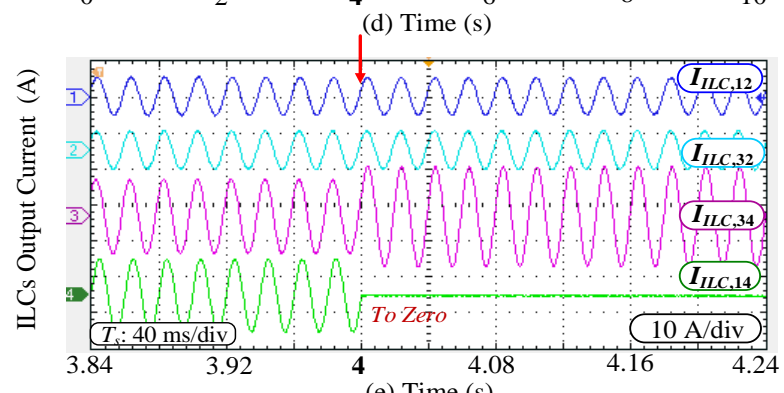

(e) Time (s)



Fig. 13. Dynamics of the system with self-optimizing power control when ILC \#14 fails at $\mathrm{t}=4 \mathrm{~s}$. (a) Active power of four subgrids, (b) active power of four ILCs, (c) reactive power of two AC subgrids, (d) reactive power of four ILCs, (e) output current of four ILCs, and (f) operation state deviation index of four subgrids.

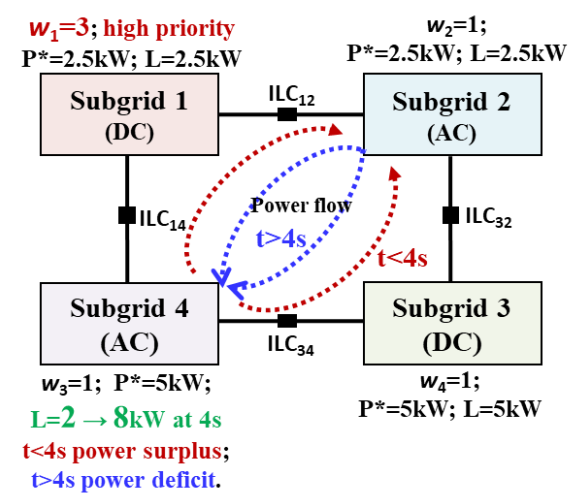

Fig. 12. A brief power flow chart of four subgrids by ILCs at different times.

three subgrids to the subgrid-4. ILC\#34 and ILC\#14 work in the inverter mode, and ILC\#12 works in the rectifier mode. On the whole, the total dispatchable energy sources are shared among the subgrids according to the priority-driven principle. Besides, the following power balance equation (20) is always ensured from Table IV.

In addition, Fig. 11 (d.1)-(e.1) show the reactive power and voltage waveforms of four ILCs. It illustrates that the quasidroop controlled ILCs not only achieve a flexible selfoptimizing power exchange flow, but ancillary functions of reactive power compensation and voltage support are also provided. Meanwhile, the output voltages of ILCs have a satisfactory dynamic response under load change.

From Case I, the proposed priority-driven self-optimizing power control method can autonomously achieve flexible power exchange management and work well in the loadchange condition.

\section{B. Case II: One ILC Unit Fault Redundancy}

This case aims to verify the one-ILC fault redundancy. Fig. 13 shows dynamics of the system with self-optimizing power control when the ILC\#14 fails at $t=4 \mathrm{~s}$. From Fig. 13(e), the output current of ILC\#14 suddenly changes to 0 at $\mathrm{t}=4 \mathrm{~s}$. Accordingly, the output active power in Fig. 13(b) and reactive power in Fig. 13(d) of ILC\#14 become 0. Because the output power of ILC \#14 becomes from $0.3 \mathrm{~kW}$ to 0 , the power flows of the other three ILCs are re-dispatched.

Although ILC\#14 fails, the output active powers of four subgrids are not affected in Fig. 13 (a), and the operation state deviation indexes of four subgrids are unchanged in Fig. 13(f), which verifies the correctness and uniqueness of prioritydriven self-optimizing solutions.

Summarily, the system can still work well after disconnecting the ILC\#14 at $\mathrm{t}=4 \mathrm{~s}$. Meanwhile, ILCs reach the new steady states after about 1 second in Fig. 13(b). As seen, the results indicate that the proposed method achieves a oneILC-unit fault redundancy.

\section{Case III: Impact of Large Difference Between Priority Weights on Transient Process}

This case is to analysis the impact of a large difference between priority weights on the transient process of the loadchange condition. Compared with the Case I, the priority weight of $w_{1}$ increases from 3 to 5 to reduce the operation state 
deviation of subgrid-1 in the Case III. Fig. 14 shows dynamics of the system with a higher $w_{1}$ when load changes at $\mathrm{t}=4 \mathrm{~s}$.

From Fig. 14(a), (c), a higher $w_{1}$ has little impact on the dynamic responses of the output active power and operation state deviation indexes of four subgrids. The transient process time of algorithm convergence is about $2 \mathrm{~s}$, wich is always satisfactory. While for Fig. 14(b), a higher $w_{1}$ causes a large overshoot of $P_{I L C, 14}$. The main reason is that droop-controlled subgrids have less-inertia, and the frequency of subgrid-4 changes instantly when the subgrid-4 load switches, as shown in Fig. 14(c). As a result, the output active power of the ILC\#14 has an instantaneous impact. In the practical microgrid with a certain inertia moment, the power impact of the ILCs would be greatly improved.
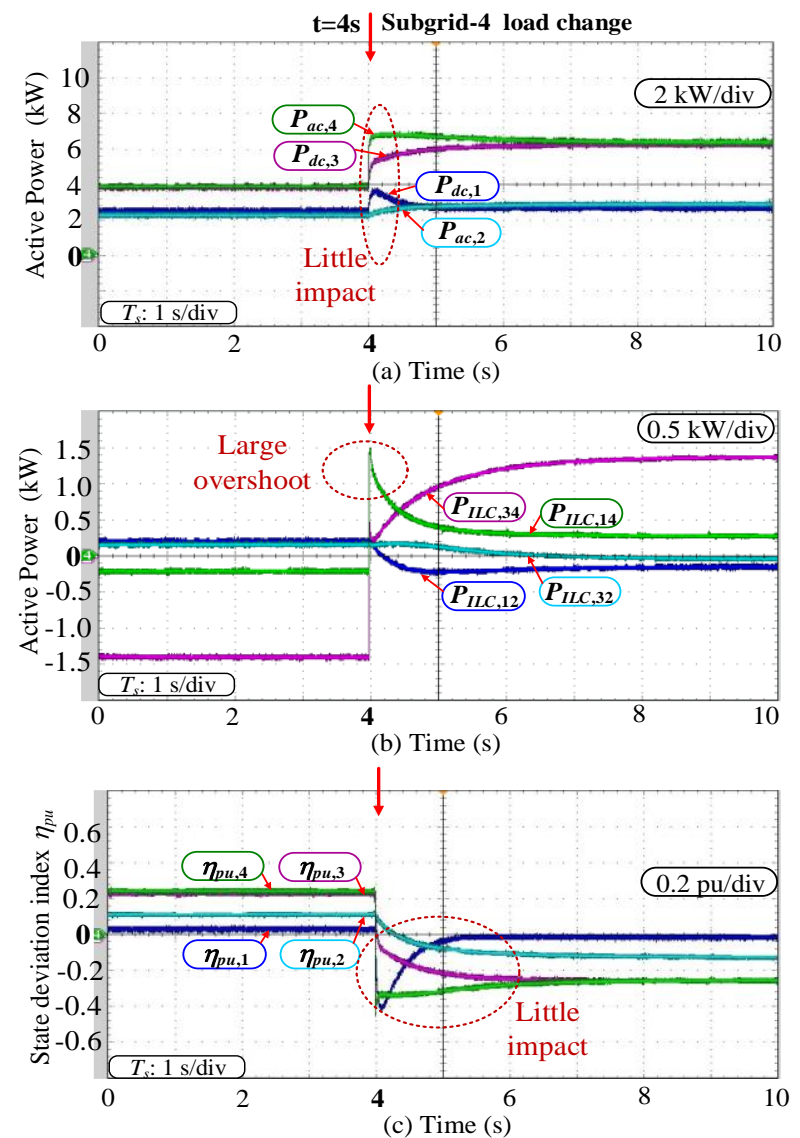

Fig. 14. Dynamics of the system with a higher priority weight $w_{1}=5$. (a) Active power of four subgrids, (b) active power of four ILCs, and (c) operation state deviation index of four subgrids.

\section{CONCLUSIONS}

To ensure the optimal power exchanges among microgrid clusters in priority, this study proposes a decentralized selfoptimizing power exchange control scheme of ILCs for hybrid microgrid clusters. For a high-priority subgrid with critical loads, its power supply and voltage quality are autonomously prioritized to realize a flexible power flow management. In the proposed strategy, the self-optimizing coordination control of ILCs is executed in a decentralized manner, which controls the power exchanges based on locally monitored AC frequency and DC voltage without a central controller or any communication. Thus, the proposed control is more costeffective, higher reliable and resilient in terms of communication faults. HIL results show the effectiveness of the proposed method with four interlinked AC/DC microgrids.

Besides, the proposed self-optimizing rule provides a new paradigm to manage complex microgrid clusters towards a decentralized self-organizing grid. It can guarantee the basic operation by avoiding communication dependency, which has some key features to address complicated problems 1) decentralized self-optimizing behavior; 2) reserved dispatchable source sharing among subgrids; 3) mutual PV/wind/load uncertainty complementarity among subgrids; 4) autonomous load shedding of low-priority subgrid; 5) oneILC-fault redundancy.

Based on this fundamental study, some novel hierarchical control schemes would be explored in future work, where the decentralized self-optimizing power control takes a backup role of primary control and initiates the power exchanges, and the upper-level superior management controller can ensure the economic operation by adjusting the power exchanges. Thus, the proposed self-optimizing method can be compatible with high-level control algorithms. On the other hand, this decentralized self-optimizing backup control can support the resilient operation under extreme events, such as the communication paralysis and central controller failure.

\section{REFERENCES}

[1] R. H. Lasseter, "Smart Distribution: Coupled Microgrids," Proceedings of the IEEE, vol. 99, no. 6, pp. 1074-1082, Jun. 2011.

[2] X. Liu, P. Wang, and P. C. Loh, "A hybrid AC/DC microgrid and its coordination control," IEEE Trans. Smart Grid, vol. 2, no. 2, pp. 278286, 2011.

[3] X. Lu, J. M. Guerrero, K. Sun, J. C. Vasquez, R. Teodorescu, and L. Huang, "Hierarchical Control of Parallel AC-DC Converter Interfaces for Hybrid Microgrids," IEEE Trans. Smart Grid, vol. 5, no. 2, pp. 683-692, Mar. 2014.

[4] S. K. Sahoo, A. K. Sinha and N. K. Kishore, "Control Techniques in AC, DC, and Hybrid AC-DC Microgrid: A Review," IEEE J. Emerg. Sel. Topics Power Electron., vol. 6, no. 2, pp. 738-759, Jun. 2018.

[5] K. Y. Yap, C. M. Beh and C. R. Sarimuthu, "Fuzzy logic controllerbased synchronverter in grid-connected solar power system with adaptive damping factor," Chin. J. Elect. Eng., vol. 7, no. 2, pp. 37-49, Jun. 2021.

[6] G. Lin, W. Zuo, Y. Li, J. Liu, S. Wang, P. Wang, "Comparative Analysis on the Stability Mechanism of Droop Control and VID Control in DC Microgrid" Chin. J. Elect. Eng., vol.7, no.1, pp. 37-46, Mar. 2021.

[7] X. Shen, D. Tan, Z. Shuai, and A. Luo, "Control Techniques for Bidirectional Interlinking Converters in Hybrid Microgrids: Leveraging the advantages of both ac and dc," IEEE Power Electronics Magazine, vol. 6, no. 3, pp. 39-47, 2019.

[8] F. Nejabatkhah and Y. W. Li, "Overview of Power Management Strategies of Hybrid AC/DC Microgrid," IEEE Trans. Power Electron., vol. 30, no. 12, pp. 7072-7089, Dec. 2015.

[9] A. Gupta, S. Doolla, and K. Chatterjee, "Hybrid AC-DC Microgrid: Systematic Evaluation of Control Strategies," IEEE Trans. Smart Grid, vol. 9, no. 4, pp. 3830-3843, 2018.

[10] J. Wang and X. Lu, "Sustainable and Resilient Distribution Systems With Networked Microgrids," Proceedings of the IEEE, vol. 108, no. 2, pp. 238-241, Feb. 2020.

[11] Z. Li, M. Shahidehpour, F. Aminifar, A. Alabdulwahab and Y. AlTurki, "Networked Microgrids for Enhancing the Power System Resilience," Proceedings of the IEEE, vol. 105, no. 7, pp. 1289-1310, Jul. 2017.

[12] B. Chen, J. Wang, X. Lu, C. Chen and S. Zhao, "Networked Microgrids for Grid Resilience, Robustness, and Efficiency: A Review," IEEE Trans. Smart Grid, vol. 12, no. 1, pp. 18-32, Jan. 2021.

[13] K. Sun, X. Wang, Y. W. Li, F. Nejabatkhah, Y. Mei, and X. Lu, "Parallel Operation of Bidirectional Interfacing Converters in a Hybrid 
AC/DC Microgrid Under Unbalanced Grid Voltage Conditions," IEEE Trans. Power Electron., vol. 32, no. 3, pp. 1872-1884, Mar. 2017.

[14] P. Wang, C. Jin, D. Zhu, Y. Tang, P. C. Loh and F. H. Choo, "Distributed Control for Autonomous Operation of a Three-Port AC/DC/DS Hybrid Microgrid," IEEE Trans. Ind. Electron., vol. 62, no. 2, pp. 1279-1290, Feb. 2015.

[15] L. Meng, F. Tang, M. Savaghebi, J. C. Vasquez and J. M. Guerrero, "Tertiary Control of Voltage Unbalance Compensation for Optimal Power Quality in Islanded Microgrids," IEEE Trans. Energy Conversion, vol. 29, no. 4, pp. 802-815, Dec. 2014.

[16] Y. Zhang, L. Xie and Q. Ding, "Interactive Control of Coupled Microgrids for Guaranteed System-Wide Small Signal Stability," IEEE Trans. Smart Grid, vol. 7, no. 2, pp. 1088-1096, Mar. 2016.

[17] Q. Zhou, M. Shahidehpour, A. Alabdulwahab and A. Abusorrah, "Flexible Division and Unification Control Strategies for Resilience Enhancement in Networked Microgrids," IEEE Trans. Power Systems, vol. 35, no. 1, pp. 474-486, Jan. 2020.

[18] G. Qi, "Improved Control Strategy of Interlinking Converters With Synchronous Generator Characteristic in Islanded Hybrid AC/DC Microgrid," CPSS Trans. Power Electronics and Applications, vol. 2, no. 2, pp. 149-158, Jun. 2017.

[19] X. Li, L. Guo, Y. Li, Z. Guo, C. Hong, Y. Zhang, and C. Wang, "A unified control for the DC-AC interlinking converters in hybrid AC/DC Microgrids," IEEE Trans. Smart Grid, vol. 9, no. 6, pp. 6540-6553, 2018.

[20] J. Wang, C. Jin, and P. Wang, "A Uniform Control Strategy for the Interlinking Converter in Hierarchical Controlled Hybrid AC/DC Microgrids," IEEE Trans. Ind. Electron., vol. 65, no. 8, pp. 6188-6197, Aug. 2018

[21] J. Li, Y. Liu and L. Wu, "Optimal Operation for Community-Based Multi-Party Microgrid in Grid-Connected and Islanded Modes," IEEE Trans. Smart Grid, vol. 9, no. 2, pp. 756-765, Mar. 2018.

[22] V. Bui, A. Hussain and H. Kim, "A Strategy for Flexible Frequency Operation of Stand-Alone Multimicrogrids," IEEE Trans. Sustainable Energy, vol. 9, no. 4, pp. 1636-1647, Oct. 2018

[23] Z. Wang, B. Chen, J. Wang, M. M. Begovic and C. Chen, "Coordinated Energy Management of Networked Microgrids in Distribution Systems," IEEE Trans. Smart Grid, vol. 6, no. 1, pp. 45-53, Jan. 2015.

[24] P. Lin, P. Wang, C. Jin, J. Xiao, X. Li, F. Guo, and C. Zhang, "A Distributed Power Management Strategy for Multi-Paralleled Bidirectional Interlinking Converters in Hybrid AC/DC Microgrids," IEEE Trans. Smart Grid, vol. 10, no. 5, pp. 5696-5711, 2019.

[25] P. Lin, C. Jin, J. Xiao, X. Li, D. Shi, Y. Tang, and P. Wang, "A distributed control architecture for global system economic operation in autonomous hybrid ac/dc microgrids," IEEE Trans. Smart Grid, vol. 10, no. 3, pp. 2603-2617, 2019.

[26] Q. Zhou, Z. Li, Q. Wu and M. Shahidehpour, "Two-Stage Load Shedding for Secondary Control in Hierarchical Operation of Islanded Microgrids," IEEE Trans. Smart Grid, vol. 10, no. 3, pp. 3103-3111, May 2019.

[27] C. Jin, P. C. Loh, P. Wang, Y. Mi, and F. Blaabjerg, "Autonomous operation of hybrid AC-DC microgrids," 2010 IEEE Int. Conf. Sustain. Energy Technol. ICSET 2010, 2010.

[28] P. Ch. Loh, D. Li, Y. K. Chai, and F. Blaabjerg, "Autonomous Operation of Hybrid Microgrid with AC and DC Subgrids", IEEE Trans. Power Electron., vol. 28, no. 5, pp. 2214-2223, May. 2013.

[29] P. Ch. Loh, D. Li, Y. K. Chai, and F. Blaabjerg, "Autonomous Control of Interlinking Converter With Energy Storage in Hybrid AC-DC Microgrid", IEEE Trans. Ind. App., vol. 49, no. 3, pp. 1374-1383, May 2013.

[30] S. Malik, Y. Sun, W. Huang, X. Ai, and Z. Shuai, "A Generalized Droop Strategy for Interlinking Converter in a Standalone Hybrid Microgrid," Applied Energy, vol. 226, pp. 1056-1063, 2018.

[31] Y. Xia, W. Wei, M. Yu, Y. Peng and J. Tang, "Decentralized MultiTime Scale Power Control for a Hybrid AC/DC Microgrid With Multiple Subgrids," IEEE Trans. Power Electron., vol. 33, no. 5, pp. 4061-4072, May 2018.

[32] Q. Xu, J. Xiao, P. Wang and C. Wen, "A Decentralized Control Strategy for Economic Operation of Autonomous AC, DC, and Hybrid AC/DC Microgrids," IEEE Trans. Energy Conversion, vol. 32, no. 4, pp. 1345-1355, Dec. 2017

[33] F. Guo, C. Wen, J. Mao, J. Chen and Y. Song, "Hierarchical Decentralized Optimization Architecture for Economic Dispatch: A New Approach for Large-Scale Power System," IEEE Trans. Industrial Informatics, vol. 14, no. 2, pp. 523-534, Feb. 2018.
[34] M. Tahir and S. K. Mazumder, "Event- and Priority-Driven Coordination in Next-Generation Grid," IEEE J. Emerg. Sel. Topics Power Electron., vol. 4, no. 4, pp. 1186-1194, Dec. 2016.

[35] J. Li, Y. Liu and L. Wu, "Optimal Operation for Community-Based Multi-Party Microgrid in Grid-Connected and Islanded Modes," IEEE Trans. Smart Grid, vol. 9, no. 2, pp. 756-765, Mar. 2018.

[36] B. Kroposki et al., "Autonomous Energy Grids: Controlling the Future Grid With Large Amounts of Distributed Energy Resources," IEEE Power and Energy Magazine, vol. 18, no. 6, pp. 37-46, Nov.-Dec. 2020.

[37] W. Yuan, Y. Wang, D. Liu, F. Deng and Z. Chen, "EfficiencyPrioritized Droop Control Strategy of AC Microgrid," IEEE J. Emerg. Sel. Topics Power Electron., vol. 9, no. 3, pp. 2936-2950, Jun. 2021.

[38] T. Ma, J. Wu, and X. Niu, "Reliability assessment indices and method for urban microgrid," in Proc. 24th International Conference and Exhibition on Electricity Distribution (CIRED), Glasgow, United Kingdom, Jun. 2017.

[39] K. N. Kumar, B. Sivaneasan and P. L. So, "Impact of Priority Criteria on Electric Vehicle Charge Scheduling," IEEE Trans. Transportation Electrification, vol. 1, no. 3, pp. 200-210, Oct. 2015.

[40] J. W. Simpson-Porco, F. Dörfler, and F. Bullo, "Synchronization and power sharing for droop-controlled inverters in islanded microgrids," Automatica, vol. 49, no. 9, pp. 2603-2611, Sep. 2013.

[41] S. Bhattacharya and S. Mishra, "Stability enhancement of droop controlled PV based Microgrid with frequency dependent load," 2014 North Am. Power Symp. NAPS 2014, pp. 0-5, 2014.

[42] A. Kahrobaeian and Y. A.-R. I. Mohamed, "Analysis and Mitigation of Low-Frequency Instabilities in Autonomous Medium-Voltage Converter-Based Microgrids With Dynamic Loads," IEEE Trans. Ind. Electron.,vol. 61, no. 4, pp. 1643-1658, Apr. 2014.

[43] X. Zhang, Q. Gao, Y. Hu, H. Zhang, and Z. Guo, "Active power reserve photovoltaic virtual synchronization control technology," Chin. J. Elect. Eng., vol. 6, no. 2, pp. 1-6, Jun. 2020.

[44] W. Wu et al., "Sequence Impedance Modeling and Stability Comparative Analysis of Voltage-Controlled VSGs and CurrentControlled VSGs," IEEE Trans. Industrial Electronics, vol. 66, no. 8, pp. 6460-6472, Aug. 2019.

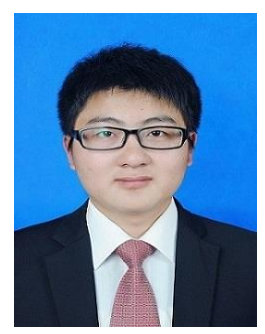

Xiaochao Hou (S'16-M'20) received the B.S., M.S., and $\mathrm{Ph} . \mathrm{D}$. degrees in control science and engineering from the School of Automation, Central South University, Changsha, China, in 2014, 2017, and 2020, respectively. From Sep. 2018 to Sep. 2019, he was a joint Ph.D. student with the School of Electrical and Electronic Engineering of Nanyang Technological University, Singapore. $\mathrm{He}$ is currently working as Postdoctoral Research Fellow in Tsinghua University, China. His research interests include control and stability of distributed micro-grid and series/parallel-type power network.

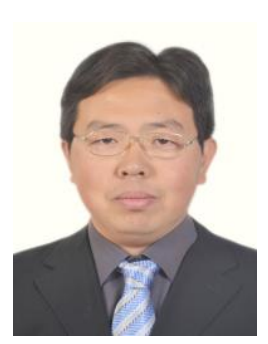

Kai Sun (M'12-SM'16) received the B.E., M.E., and $\mathrm{Ph} . \mathrm{D}$. degrees in electrical engineering from Tsinghua University, in 2000, 2002, and 2006, respectively. He joined the faculty of Electrical Engineering, Tsinghua University, in 2006, where he is currently a Tenured Associate Professor (Research Professor). From Sep 2009 to Aug 2010, he was a Visiting Scholar at Department of Energy Technology, Aalborg University, Aalborg, Denmark. From Jan to Aug 2017, he was a Visiting Professor at Department of Electrical and Computer Engineering, University of Alberta, Edmonton, Canada. His research interests include power electronics for renewable generation systems, microgrids, and energy internet.

Dr. Sun serves as an Associate Editor for IEEE Transactions on Power Electronics, IEEE Journal of Emerging and Selected Topics in Power Electronics, and Journal of Power Electronics. Dr. Sun served as the TPC Vice Chair of IEEE ECCE2017 and IEEE ECCE-Asia2017, the Organization Committee Chair of IEEE eGrid2019, and the Publicity Chair of IEEE ECCE2020. He also served as the General Co-Chair of 2018 International Future Energy Challenge (IFEC2018). Dr. Sun serves as PELS Asia Pacific Regional Vice Chair, PELS Beijing Chapter Chair and PELS Electronic 
Power Grid Systems Technical Committee (TC8) Secretary. He was a recipient of Delta Young Scholar Award in 2013, and Youth Award of China Power Supply Society (CPSS) in 2017, and IEEE Transactions on Power Electronics' Outstanding Reviewers Award in 2019. Dr. Sun is selected as IEEE PELS Distinguished Lecturer in 2021-2022.

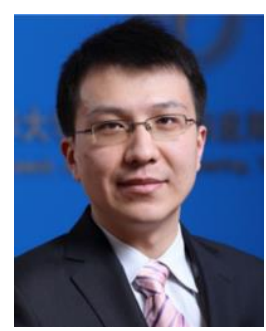

Ning Zhang (Senior Member, IEEE) received the B.S. and Ph.D. degrees in in electrical engineering from Tsinghua University, Beijing, China, in 2007 and 2012, respectively.

$\mathrm{He}$ is currently an Associate Professor with Tsinghua University. His research interests include multiple energy systems integration, renewable energy, and power system planning and operation.

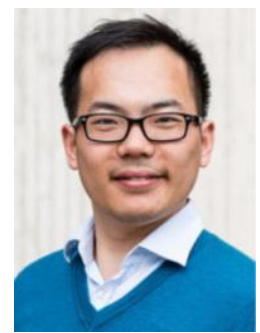

Fei Teng (Senior Member, IEEE) received the B.Eng. degree in electrical engineering from Beihang University, China, in 2009, and the Ph.D. degree in electrical engineering from Imperial College London, U.K., in 2015.

$\mathrm{He}$ is currently a Lecturer with the Department of Electrical and Electronic Engineering, Imperial College London. His research focuses on scheduling and market design for low-inertia power system, cyber-resilient energy system operation and control, and objective-based data analytics for future energy

systems.

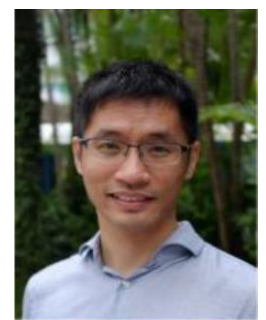

Xin Zhang (M'15-SM'20) received the Ph.D. degree in Automatic Control and Systems Engineering from the University of Sheffield, U.K., in 2016 and the $\mathrm{Ph} . \mathrm{D}$. degree in Electronic and Electrical Engineering from Nanjing University of Aeronautics \& Astronautics, China, in 2014. From 2017 to 2020, he was an Assistant Professor of Power Engineering at the School of Electrical and Electronic Engineering of Nanyang Technological University, Singapore. Currently, he is Research Professor at College of Electrical Engineering, Zhejiang University, China. He was the Postdoctoral Research Fellow (2017.01 - 2017.09) at the City University of Hong Kong and the Research Associate (2014.02 - 2016.12) at the University of Sheffield. He is generally interested in power electronics, power system, and advanced control theory, together with their applications in various sectors.



Tim C. Green (Fellow, IEEE) received the B.Sc. (Eng) (first class honours) from Imperial College London, U.K. in 1986 and a Ph.D. degree from HeriotWatt University, Edinburgh, U.K. in 1990. $\mathrm{He}$ is a Professor of electrical power engineering at Imperial College London, and Director of the Energy Futures Lab with a role fostering interdisciplinary energy research. His research interest is in using the flexibility of power electronics to accommodate new generation patterns and new forms of load, such as EV charging, as part of the emerging smart grid. In HVDC he has contributed converter designs that reduce losses while also providing control functions assist AC system integration. In distribution systems, he has pioneered the use of soft open points and the study of stability of grid connected inverters. Prof. Green is a Chartered Engineering the U.K. and a Fellow of the Royal Academy of Engineering. 